\title{
Mitochondrial Dysfunction and Defective Autophagy in the Pathogenesis of Collagen VI Muscular Dystrophies
}

\author{
Paolo Bernardi and Paolo Bonaldo \\ Department of Biomedical Sciences, University of Padova, I-35121 Padova, Italy \\ Correspondence: bernardi@bio.unipd.it; bonaldo@bio.unipd.it
}

Ullrich Congenital Muscular Dystrophy (UCMD), Bethlem Myopathy (BM), and Congenital Myosclerosis are diseases caused by mutations in the genes encoding the extracellular matrix protein collagen VI. A dystrophic mouse model, where collagen VI synthesis was prevented by targeted inactivation of the Col6a1 gene, allowed the investigation of pathogenesis, which revealed the existence of a $\mathrm{Ca}^{2+}$-mediated dysfunction of mitochondria and sarcoplasmic reticulum, and of defective autophagy. Key events are dysregulation of the mitochondrial permeability transition pore, an inner membrane high-conductance channel that for prolonged open times causes mitochondrial dysfunction, and inadequate removal of defective mitochondria, which amplifies the damage. Consistently, the Col6a1 $1^{-1-}$ myopathic mice could be cured through inhibition of cyclophilin D, a matrix protein that sensitizes the pore to opening, and through stimulation of autophagy. Similar defects contribute to disease pathogenesis in patients irrespective of the genetic lesion causing the collagen VI defect. These studies indicate that permeability transition pore opening and defective autophagy represent key elements for skeletal muscle fiber death, and provide a rationale for the use of cyclosporin $\mathrm{A}$ and its nonimmunosuppressive derivatives in patients affected by collagen VI myopathies, a strategy that holds great promise for treatment.

\begin{abstract}
A nchoring and adhesion complexes at the Asurface of cells link the cytoskeleton to the surrounding extracellular matrix (ECM), thus maintaining cell integrity and allowing signal transduction. These anchoring structures have a critical role in tissues undergoing extensive mechanical stress, like skeletal muscle; it comes as no coincidence that genetic defects of components of the anchoring complexes cause human muscular dystrophies. The ECM is directly involved in the molecular pathogenesis of vari-
\end{abstract}

ous forms of muscular dystrophy, and there is accumulating evidence that ECM components, such as laminin-2 and collagen VI (ColVI), play a critical role in maintaining muscle integrity and function (Schessl et al. 2006).

ColVI is a main ECM protein forming a distinct microfilamentous network with a broad distribution in several organs including skeletal muscle, skin, cornea, lung, blood vessels, intervertebral disks, and joints (Keene et al. 1988). It consists of three major chains, $\alpha 1(\mathrm{VI})$,

Editors: Douglas C. Wallace and Richard J. Youle

Additional Perspectives on Mitochondria available at www.cshperspectives.org

Copyright (C) 2013 Cold Spring Harbor Laboratory Press; all rights reserved; doi: 10.1101/cshperspect.a011387 Cite this article as Cold Spring Harb Perspect Biol 2013;5:a011387 
$\alpha 2(\mathrm{VI})$, and $\alpha 3(\mathrm{VI})$, encoded by separate genes (COL6A1, COL6A2, and COL6A3, respectively). The $140-\mathrm{kD} \alpha 1(\mathrm{VI})$ and $\alpha 2(\mathrm{VI})$ chains are about 1000 amino acids long, while the $\alpha 3(\mathrm{VI})$ chain is three times larger $(250-300 \mathrm{kD})$ with several alternatively spliced variants ranging between $\sim 2500$ and 3100 amino acids (Bonaldo et al. 1989, 1990; Chu et al. 1990; Doliana et al. 1990). ColVI is a multimodular protein made of several domains; each chain contains a short triple helical domain of 335-336 amino acids and two large amino- and carboxy-terminal globular ends composed of repeated domains of 200 amino acids each, sharing similarity with the von Willebrand factor type A (vWF-A) module (Colombatti and Bonaldo 1991). Three additional ColVI chains, $\alpha 4(\mathrm{VI}), \alpha 5(\mathrm{VI})$, and $\alpha 6(\mathrm{VI})$, were recently identified. These chains, coded by distinct genes (COL6A4-COL6A6), show a high degree of similarity with $\alpha 3(\mathrm{VI})$, and their tissue distribution is more restricted, suggesting a specific role in the assembly and/or function of ColVI (Gara et al. 2008, 2011; Sabatelli et al. 2011).

ColVI has a complex pathway of intracellular assembly (Colombatti and Bonaldo 1987; Colombatti et al. 1987, 1995). Association of the $\alpha$-chains into a triple helical monomer (three chains) is followed by formation of dimers (six chains) and tetramers (12 chains), stabilized by disulfide bonds. After secretion into the extracellular space, the tetramers interact in an endto-end fashion and form "beaded" microfilaments with a typical 100-nm periodicity, which are deposited into the ECM (Furthmayr et al. 1983). ColVI microfilaments form an extended network particularly abundant in the close periphery of several cell types, and they have been suggested to bridge the surface of cells with the interstitial connective tissue (Keene et al. 1988; Bonaldo et al. 1990; Kuo et al. 1997). In skeletal muscle, ColVI is a major component of endomysium, where it is localized in the outer layer of the basement membrane (Kuo et al. 1997).

The different domains of ColVI are involved in a broad range of interactions with cell surface receptors and with other ECM components. The triple helical region of ColVI is a major cell-binding domain, and it promotes adhe- sion through interaction with several integrins and with NG2 proteoglycan (Pfaff et al. 1993; Burg et al. 1996). Both the triple helical region and the vWF-A domains of ColVI bind various ECM proteins including fibrillar collagens, basal lamina (type IV) collagen, decorin, biglycan, and fibronectin (Bonaldo et al. 1990; Bidanset et al. 1992; Kuo et al. 1997; Sabatelli et al. 2001; Wiberg et al. 2001). These interactions and the close association of beaded microfilaments with muscle basal lamina strongly suggest that ColVI provides an important mechanical link of muscle cells to the surrounding ECM.

ColvI is synthesized and secreted by cells organizing an ECM, such as fibroblasts and muscle cells. Studies in transfected cells and in transgenic mice demonstrated that transcriptional regulation is the major determinant for the highly regulated and dynamic pattern expression of ColVI during development and in adult tissues. The data thus far available for the Col6a1 gene indicate a complex pattern of tissue-specific transcription that is dependent on different enhancer and silencer elements spread over a large genomic region and controlling expression in skin, tendons, joints, peripheral nerves, and skeletal muscles, respectively (Braghetta et al. 1996). Muscle interstitial fibroblasts are the main source of ColVI in the ECM of skeletal muscle (Zou et al. 2008). A study revealed that transcription of the Col6a1 gene in skeletal muscle is under the control of a musclespecific enhancer, which is strictly required for activating the expression and synthesis of ColVI in muscle fibroblasts (Braghetta et al. 2008). Remarkably, activation of this enhancer requires signals relayed by myogenic cells, whose presence is a prerequisite for inducing the deposition of ColVI by interstitial fibroblasts (Braghetta et al. 2008). In vitro and in vivo studies have shown that ColVI is synthesized by interstitial fibroblasts; once released in the ECM, the protein contacts myogenic cells and myofibers (Zou et al. 2008; Palma et al. 2009).

\section{COLLAGEN VI-RELATED MUSCLE DISEASES}

Deficiency of ColVI in humans gives rise to three main syndromes, Bethlem myopathy 
(BM), Ullrich congenital muscular dystrophy (UCMD) (Lampe and Bushby 2005; Bönnemann 2011), and the recently identified congenital myosclerosis (Merlini et al. 2008b).

BM (MIM \#158810; http://www.metalife. com/OMIM) is characterized by axial and proximal muscle weakness with finger joint contractures, proximal muscles being more involved than distal, and extensors more than flexors (Bethlem and Wijngaarden 1976). The hallmark of the disease is the presence of contractures of the interphalangeal joints of the last four fingers, and early flexion contractures of the elbow and ankles are common (Merlini et al. 1994). BM is a very heterogeneous disorder, and patients show a wide range of clinical features, from mild myopathy to more severe cases with early onset and features of slowly progressive muscular dystrophy. BM inheritance is usually autosomal dominant (Jöbsis et al. 1996; Sasaki et al. 2000; Scacheri et al. 2002), but recessive cases were also described (Foley et al. 2009; Gualandi et al. 2009). Immunohistochemistry shows apparently normal or mildly reduced levels of ColVI in the endomysium of most BM patients, and quantitative or qualitative ColVI defects can be detected in cultured fibroblasts derived from skin biopsies.

UCMD (MIM \#254090) is a severe congenital muscular dystrophy characterized by early onset, generalized and rapidly progressive muscle wasting and weakness, proximal joint contractures, and distal joint hyperflexibility. The rapid progression of the clinical symptoms usually leads to early death, as a result of respiratory failure (Camacho Vanegas et al. 2001; Demir et al. 2002). Usually, UCMD shows an autosomal recessive inheritance with homozygous or compound heterozygous mutations of the COL6 genes, but several cases of UCMD with dominant mutation in only one allele were also reported (Pan et al. 2003; Baker et al. 2005; Angelin et al. 2007). Patients with a UCMD phenotype but without mutations in COL6 genes were described, suggesting a possible genetic heterogeneity for the disease. ColVI appears to be strongly reduced or absent in muscle biopsies from UCMD patients, suggesting that UCMD mutations severely affect the synthesis and se- cretion of ColVI in muscle. Cultured skin fibroblasts of UCMD patients usually show either a markedly decreased secretion of ColVI, or lack of the characteristic filamentous network in the ECM, suggesting that UCMD mutations severely affect the synthesis and secretion of ColVI (Camacho Vanegas et al. 2001; Zhang et al. 2002; Squarzoni et al. 2006).

Congenital myosclerosis (MIM \#255600) is a rare inherited disorder characterized by slender muscles with firm "woody" consistency, and restricted movement of many joints because of diffuse muscle contractures. Reduced expression of ColVI was detected in muscle biopsies and cultured fibroblasts from affected sibs of an Italian family, and the disease is linked to a homozygous mutation of the COL6A2 gene (Merlini et al. 2008b).

Mutations in any of the COL6A1-COL6A3 genes have been identified in BM and in UCMD (Lampe and Bushby 2005; Bönnemann 2011). Different COL6 gene mutations may have different impacts on the mRNA and protein level by predominantly impairing ColVI synthesis, assembly, secretion, or function. Based on the mutation, different mechanisms can be predicted such as: (1) loss-of-function for mutations perturbing triple helix formation, intracellular assembly or ColVI secretion; (2) dominant negative effect for mutations giving rise to abnormal ColVI polypeptides secreted in the ECM; and (3) haploinsufficiency for mutations affecting mRNA stability, with a decreased synthesis of normal ColVI. The effect of specific COL6 mutations has been investigated by in vitro studies (Lamande et al. 1999, 2002; Sasaki et al. 2000; Zhang et al. 2002; Jimenez-Mallebrera et al. 2006; Baker et al. 2007; Tooley et al. 2010), which provided relevant clues on their effect at the protein level. However, there is no conclusive evidence on the genotype-phenotype correlation for BM and UCMD, which may represent a clinical continuum rather than strictly separate entities (Pepe et al. 2002; Lampe and Bushby 2005; Bönnemann 2011).

As for many other genetic diseases, creation of animal models of ColVI deficiency proved to be the key to understanding disease pathophysiology, and to devise and test potential 
therapies. Several years ago, one of us produced a mutant mouse with targeted inactivation of the Col6al gene, which codes for the $\alpha 1(\mathrm{VI})$ chain (Bonaldo et al. 1998). In the absence of the $\alpha 1$ chain, ColVI does not assemble and is not secreted in the ECM, so that Colba1 ${ }^{-/-}$ mice completely lack ColVI and are affected by a myopathic phenotype with weakness and histological changes of skeletal muscle, characterized by early onset and slow or no progression (Bonaldo et al. 1998). These defects are found in various skeletal muscles, in particular the diaphragm, as revealed by in vivo Evans blue dye staining. Similar but milder defects are present in $\mathrm{Col}_{6 a 1}{ }^{+/-}$mice, indicating functional haploinsufficiency for ColVI. Muscle weakness was confirmed by strength measurements ex vivo, showing significantly reduced tension in Colba1 ${ }^{-1-}$ muscles (Irwin et al. 2003). Although Col6a1-null mice are a model of human ColVI diseases, their phenotype is much closer to the benign myopathy of BM than to the severe dystrophic pathology of UCMD. A recent advance has been the generation of zebrafish models of UCMD and BM, which reproduce the key features of the human diseases (Telfer et al. 2010).

\section{PATHOGENESIS OF COLLAGEN VI MUSCULAR DYSTROPHIES}

Ultrastructural analysis of skeletal muscle from Colba1 ${ }^{-/-}$mice revealed the presence of marked dilations of the sarcoplasmic reticulum (SR) and of mitochondrial alterations in $20 \%-$ $30 \%$ of the fibers, while T-tubules were normal (Irwin et al. 2003). Mitochondrial abnormalities ranged from tubular cristae, to electron-dense matrix inclusions, to swelling; myofibers with mitochondrial-SR alterations also displayed nuclear features of apoptosis, suggesting a link between organelle changes and increased incidence of cell death (Irwin et al. 2003). Mitochondrial ultrastructural alterations are observed in many forms of muscle pathology; whether these are the cause or consequence of the disease (or even a side effect of no pathogenic relevance) was the key question that needed to be answered.
Mitochondria in the Pathogenesis of Collagen VI Muscular Dystrophies

We monitored the mitochondrial membrane potential in skeletal muscle fibers and myoblast/fibroblast cultures of Col6a1 $1^{-/-}$and wild-type mice, and in muscle cell cultures from patients affected by ColVI myopathies, or from normal donors. The resting mitochondrial membrane potential was not affected by lack of ColVI, yet mitochondria depolarized after addition of the F1FO ATP synthase inhibitor oligomycin or of the respiratory chain complex I inhibitor rotenone only in cells from ColVIdeficient individuals (Irwin et al. 2003; Angelin et al. 2007, 2008; Palma et al. 2009; Tiepolo et al. 2009; Sabatelli et al. 2012). Neither response is normal because, in the short term at least, (1) inhibition of ATP synthesis by oligomycin should cause hyperpolarization, and (2) inhibition of respiration should still allow the membrane potential to be maintained by proton pumping by the ATP synthase running "in reverse" and utilizing glycolytic ATP as fuel (see, e.g., Porcelli et al. 2009). Of note, cells from ColVI-defective genotypes also displayed increased rates of apoptosis that could be prevented by plating on ColVI or by addition of cyclosporin A (CsA), treatments that also normalized the mitochondrial response to oligomycin and/ or rotenone (Irwin et al. 2003; Angelin et al. 2007, 2008; Palma et al. 2009; Tiepolo et al. 2009; Sabatelli et al. 2012). These findings, which we shall discuss further below, (1) suggest the existence of a latent (compensated) mitochondrial dysfunction that may worsen (and decompensate) with time, which is consistent with the chronic and progressive features of ColVI muscular dystrophies; and (2) indicate that the underlying mitochondrial dysfunction, and possibly the disease, can be cured with CsA.

The pharmacology of CsA is complex. This drug binds to and inhibits cyclophilins ( $\mathrm{CyPs}$ ), a family of cellular peptidyl-prolyl cis-trans isomerases (Galat 2003). The complex of CsA with the abundant cytosolic CyPA acquires the ability to bind and inhibit the cytosolic phosphatase calcineurin, preventing the dephosphorylation of its substrates (Liu et al. 1991), 
which include NFAT and Drp1. Dephosphorylation is essential for the nuclear translocation of NFAT, which is key to activation of the T lymphocytes involved in the immune response to organ transplants; by preventing NFAT nuclear translocation, CsA prevents transplant rejection (Liu et al. 1991). Dephosphorylation of Drp1 is essential for its translocation to mitochondria, where it causes fission and fragmentation, events that are prevented by CsA (Cereghetti et al. 2008). Neither effect is involved in the protective mitochondrial effects of CsA in ColVI diseases because the rescue from mitochondrial dysfunction cannot be mimicked by the calcineurin inhibitor FK506 (Irwin et al. 2003), which acts through its specific receptor FKBP but does not bind CyPs (Liu et al. 1991). As we originally suggested (Irwin et al. 2003), the protective effects of CsA are rather a result of inhibition of $\mathrm{CyPD}$, the unique mitochondrial isoform of CyPs (encoded in the mouse by the Ppifgene). Indeed, the protective effects can also be seen with CsA analogs that inhibit CyPs but not calcineurin, like MeAla ${ }^{3} \mathrm{EtVal}^{4}$-cyclosporin (Debio 025) (Angelin et al. 2007; Tiepolo et al. 2009). Col6a1 ${ }^{-/-}$Ppif $^{-/-}$mice lacking both ColVI and CyPD display a striking recovery of both the mitochondrial lesions and the muscle defects (Palma et al. 2009).

The most convincing explanation for the protective effects of CsA and Debio 025 (and of CyPD ablation) in ColvI myopathies is desensitization of the permeability transition pore (PTP), a high-conductance inner membrane mitochondrial channel of unknown molecular identity involved in several forms of cell death (Bernardi et al. 2006; Rasola and Bernardi 2011). CyPD favors PTP opening, and matrix $\mathrm{Ca}^{2+}$ is an important permissive factor sensitizing the PTP to a variety of inducing factors. The pore is modulated by the proton electrochemical gradient, both through the voltage (depolarization favors opening) and $\mathrm{pH}$ (matrix acidification favors closure), and is finely tuned by the redox poise (a more oxidized state favors pore opening). A detailed coverage of PTP regulation is beyond the scope of this review, and we refer the reader to previous publications on the topic (Bernardi et al. 2006; Rasola and
Bernardi 2011). The important points to be considered here are that: (1) short-lived PTP openings cause reversible mitochondrial depolarization (Hüser and Blatter 1999; Petronilli et al. 1999) and may be involved in physiological $\mathrm{Ca}^{2+}$ homeostasis (Bernardi and von Stockum 2012); under these conditions, PTP-dependent cytochrome $c$ release can be an indirect consequence of cristae remodeling (Scorrano et al. 2002) and matrix swelling (Petronilli et al. 1994) that cooperate with tBID in the absence of outer membrane rupture (Bernardi et al. 2001); and (2) long-lasting PTP openings cause energy dissipation, release of pyridine nucleotides (Vinogradov et al. 1972; Di Lisa et al. 2001), outer membrane rupture, and massive release of cytochrome $c$ (Petronilli et al. 2001).

Our current interpretation of the mitochondrial pathogenesis of ColVI muscular dystrophies is that, initially at least, mitochondrial dysfunction is latent rather than overt, and consists in an alteration of the threshold voltage for PTP opening, which (through a still undefined mechanism that may involve matrix $\mathrm{Ca}^{2+}$ overload) is poised dangerously close to the resting potential. This shift of the voltage threshold can be unmasked in vitro by the addition of rotenone or oligomycin, which act via different mechanisms. (1) Rotenone inhibits complex I and start a process of depolarization. In normal cells, this initial depolarization can be readily compensated by the mitochondrial ATP synthase (which hydrolyzes glycolytic ATP) because the threshold voltage for PTP opening is not reached, and therefore inner membrane permeability remains low. In ColVI defective cells, the voltage threshold is so close to the resting potential that addition of rotenone causes PTP opening; ATP hydrolysis by the ATP synthase in this case cannot restore the membrane potential as long as the pore is open. (2) Oligomycin inhibits the ATP synthase, and this causes an initial hyperpolarization with parallel decrease of the ATP levels; this would cause a further shift of the threshold voltage to reach the value of the resting potential through two mechanisms, that is, increased ROS production because of the state $3 \rightarrow$ state 4 transition, and decreased ability of the SR to take up 
$\mathrm{Ca}^{2+}$, causing a secondary increase of matrix mitochondrial $\mathrm{Ca}^{2+}$. The ensuing PTP opening would then cause the observed depolarization, which is very often preceded by the expected hyperpolarization (Irwin et al. 2003; Angelin et al. 2007).

We believe that the voltage threshold shift may explain disease progression in vivo as well. Because the mitochondrial membrane potential decreases during ATP synthesis, in the ColVI defective genotypes increased PTP flickering could occur during increased muscle activity with loss of pyridine nucleotides (Vinogradov et al. 1972; Di Lisa et al. 2001) and progressive decrease of the respiratory reserve. It should be noted that ADP generated by ATP hydrolysis inhibits the PTP; thus, ATP hydrolysis may lead to pore closure and to at least partial restoration of respiration. The maximal attainable rate would be lower than normal, however, and determined by the residual matrix levels of pyridine nucleotides attained after each "cycle" of PTP opening. As long as the energy demand can be matched the fiber would behave normally, but dysfunction would be precipitated by increased workload and/or by a further increase of PTP flickering, events that will eventually lead to individual muscle fiber death as observed both in vivo and in cultured muscle fibers. The recently identified defect of autophagy would then worsen the situation by preventing removal of dysfunctional mitochondria (Grumati et al. 2010).

Defective Autophagy in the Pathogenesis of Collagen VI Muscular Dystrophies

Autophagy is a highly conserved homeostatic mechanism used for the degradation and recycling of bulk cytoplasm, long-lived proteins, and organelles through the lysosomal machinery (Mizushima et al. 2008). We recently demonstrated that autophagy plays a protective role against myofiber defects and muscle wasting in Col6a1 ${ }^{-/-}$mice. A failure of the autophagic machinery is responsible for the inefficient removal and persistence of altered organelles in myofibers of Col6a1 $1^{-/}$mice and of BM/ UCMD patients (Grumati et al. 2010). The ensuing accumulation of dysfunctional mitochondria triggers myofiber apoptosis, which in turn leads to the development of the myopathic pathology. In Col6a1-null mice, the dramatic consequences of the autophagy inactivation are even more evident in conditions of muscle stress, such as physical exercise. When Col6a1 ${ }^{-/-}$mice were subjected to an intense work, a condition in which autophagy is required for the continuous energy need and the rapid elimination of exhausted organelles, the inefficient autophagy flux determined a massive degeneration of myofibers with a marked exacerbation of the myopathic phenotype (Grumati et al. 2011b). Notably, forced activation of autophagy by dietary, genetic, and pharmacological tools was able to eliminate altered organelles and restored myofiber homeostasis in Col6a1 ${ }^{-/-}$mice, with recovery from the myopathic phenotype, thus showing a promising therapeutic potential for counteracting muscle atrophy and weakness in these diseases. The autophagic failure of Col6a1 ${ }^{-/-}$mice strictly depends on impairment of the autophagy flux, which determines a decrease in autophagosome formation and inefficient removal of dysfunctional organelles (Grumati et al. 2010). Lack of ColVI has a remarkable impact on molecules involved in the regulation of autophagy, with decreased Beclin 1 protein levels and persistent activation of the Akt/mTOR pathway, even during starvation (Grumati et al. 2010). Although the molecular pathways transducing ColVI signals from the ECM to the autophagy machinery remain to be elucidated, the Beclin 1 complex and the AKT/mTOR pathway are markedly affected by lack of ColVI, and these alterations appear to be the main cause for the autophagy inhibition. Notably, the autophagic failure of Col6a1 $^{-/-}$muscles is also associated with a decreased activity of Bnip3, a molecule playing a key role in the selective removal of damaged or dysfunctional mitochondria with a process know as "mitophagy" (Grumati et al. 2011a). Most interestingly, Beclin 1 and Bnip3 levels are also lower in muscle biopsies of patients affected by ColVI diseases, and the amount of Beclin 1 seems to correlate with the severity of the phenotype, being much lower in the severe 
UCMD than in the milder BM (Grumati et al. 2010). Our studies represented the first evidence that an impairment of the autophagic machinery plays a crucial role in the pathogenesis of muscular dystrophies, thus opening new venues for therapeutic approaches and paving the way for investigating autophagy defects in other muscular dystrophies (Tolkovsky 2010). Indeed, a recent study revealed that the $d y^{3 K} / d y^{3 K}$ mouse, which is affected by laminin $\alpha 2$ deficiency and represents a model of human MDC1A, also has an alteration of autophagy. Interestingly, at variance from Col6a1 ${ }^{-/-}$animals, $d y^{3} / d y^{3 K}$ mice displayed a general upregulation of the autophagic machinery and inhibition of autophagy significantly improved their dystrophic phenotype (Carmignac et al. 2011). This latter observation is not surprising and suggests that, as recently proposed in other pathological conditions, an appropriate balance of the autophagic flux is essential for the maintenance of skeletal muscle health.

\section{OF MICE, FISH, AND MEN}

Establishing whether the mitochondrial pathogenesis of the myopathy of Col6a1 $1^{-/-}$mice also holds for the genetically and clinically heterogeneous UCMD and BM represented a major challenge. To fill this gap, we have systematically studied muscle biopsies and cell cultures from patients affected by ColVI muscular dystrophies, and established that patients affected by UCMD and BM display an increased rate of apoptosis in skeletal muscle in vivo and in cultures derived from biopsies (Angelin et al. 2007, 2008; Merlini et al. 2008a; Sabatelli et al. 2012). The muscle cell cultures also displayed a measurable fraction of altered mitochondria, with morphological abnormalities ranging from shape changes to overt swelling, and the presence of a latent mitochondrial dysfunction that could be unmasked by the depolarizing effects of oligomycin and/or rotenone (Angelin et al. 2007, 2008; Sabatelli et al. 2012). The mitochondrial defect was present in primary cultures from UCMD and BM patients irrespective of whether the primary genetic defect was in the COL6A1 or COL6A3 gene, and both in homo- zygous and heterozygous mutations (Angelin et al. 2007), and could be rescued by both CsA and Debio 025 (Angelin et al. 2007).

Whether patient-derived cells can be safely used as a diagnostic material in ColVI diseases has recently been questioned (Hicks et al. 2009). While confirming our finding that myoblasts from patients affected by UCMD display a latent mitochondrial dysfunction that can be unmasked by oligomycin, Hicks et al. concluded that PTP dysregulation may be a particular characteristic of these cells in culture rather than being specific to the ColVI defect, because PTP dysregulation was also found in musclederived cells from patients with Limb Girdle Muscular Dystrophy (LGMD) 2B, but not BM, Merosin Deficient Congenital Muscular Dystrophy (MDC) 1A, LGMD2A, Duchenne Muscular Dystrophy (DMD), and Leigh syndrome (Hicks et al. 2009). As we already noted (Bernardi et al. 2009), mitochondria are causally involved in a variety of degenerative diseases, and it is therefore not surprising that a "mitochondrial phenotype" caused by a PTP opening can be found in other muscular dystrophies, including DMD (Millay et al. 2008; Reutenauer et al. 2008) and MDC1A (Millay et al. 2008), and in Leber hereditary optic neuropathy, a finding that extends the potential use of CsA to mitochondrial DNA diseases (Porcelli et al. 2008). The apparent discrepancy about BM was recently resolved with the demonstration that the mitochondrial phenotype of muscle cell cultures from both UCMD and BM patients was lost after nine passages in culture, which matched a spontaneous decrease of the incidence of apoptosis (Sabatelli et al. 2012). Thus, it is essential to study materials derived from patient muscle biopsies at early passages.

The mitochondrial pathogenesis of ColVI myopathies has been recently confirmed in zebrafish, an organism where the PTP has regulatory features indistinguishable from those of mammals (Azzolin et al. 2010). Injection of morpholino to exon 13 of Col6a1 caused an inframe deletion in the carboxy terminal part of the ColVI triple helical domain, a typical dominant BM mutation (Lucioli et al. 2005; Lampe et al. 2008), and resulted in a mild myopathy 
with late-onset motor deficits and obvious histopathologic abnormalities (Telfer et al. 2010). Injection of morpholino to exon 9 of Col6a1 caused an in-frame deletion in the amino terminal part of the triple helical domain, a dominant mutation in UCMD (Pepe et al. 2006; Lampe et al. 2008), and caused the predicted severe myopathy with early-onset motor deficits and severe ultrastructural changes (Telfer et al. 2010). In both cases, mitochondrial abnormalities and increased apoptosis were observed, and treatment with CsA improved these pathological signs together with rescue of the motor deficit (Telfer et al. 2010).

Based on the positive results obtained with CsA on their cells in culture (Angelin et al. 2007), a pilot trial on five patients affected by UCMD or $\mathrm{BM}$ was carried out. Prior to treatment, all patients displayed mitochondrial dysfunction and increased frequency of apoptosis, as determined in muscle biopsies. Both these pathological signs were largely normalized after 1 month of oral CsA administration, which also increased muscle regeneration (Merlini et al. 2008a). The study was then extended to six individuals with UCMD receiving 3-5 $\mathrm{mg} / \mathrm{kg}$ CsA daily for $1-$ 3.2 years (Merlini et al. 2011). The primary outcome measure was muscle strength evaluated with a myometer. The score showed significant improvement in five of the six patients, while motor function did not change and respiratory function deteriorated in all. As in the short-term treatment, CsA corrected mitochondrial dysfunction, increased muscle regeneration, and decreased the number of apoptotic nuclei (Merlini et al. 2011). These results indicate that longterm treatment with CsA is well tolerated and ameliorates performance in the limbs, but not in the respiratory muscles; they also suggest that treatment with CsA or one of its nonimmunosuppressive derivatives should be started as early as possible, when diaphragm function is not yet compromised. In summary, although much remains to be done to understand the biochemical and pathophysiological bases of ColVI diseases, we believe that the discoveries of the mitochondrial pathogenesis and autophagic defect in UCMD and BM are opening new perspectives for their therapeutic treatment.

\section{ACKNOWLEDGMENTS}

The financial support of Telethon Italy (grant GGP11082) is gratefully acknowledged.

\section{REFERENCES}

Angelin A, Tiepolo T, Sabatelli P, Grumati P, Bergamin N, Golfieri C, Mattioli E, Gualandi F, Ferlini A, Merlini L, et al. 2007. Mitochondrial dysfunction in the pathogenesis of Ullrich congenital muscular dystrophy and prospective therapy with cyclosporins. Proc Natl Acad Sci 104: $991-996$

Angelin A, Bonaldo P, Bernardi P. 2008. Altered threshold of the mitochondrial permeability transition pore in Ullrich congenital muscular dystrophy. Biochim Biophys Acta Bioenergetics 1777: 893-896.

Azzolin L, Basso E, Argenton F, Bernardi P. 2010. Mitochondrial $\mathrm{Ca}^{2+}$ transport and permeability transition in zebrafish (Danio rerio). Biochim Biophys Acta 1797: 1775-1779.

Baker NL, Morgelin M, Peat R, Goemans N, North KN, Bateman JF, Lamande SR. 2005. Dominant collagen VI mutations are a common cause of Ullrich congenital muscular dystrophy. Hum Mol Genet 14: 279-293.

Baker NL, Morgelin M, Pace RA, Peat RA, Adams NE, Gardner RJ, Rowland LP, Miller G, De JP, Ceulemans B, et al. 2007. Molecular consequences of dominant Bethlem myopathy collagen VI mutations. Ann Neurol 62: 390-405.

Bernardi P, von Stockum S. 2012. The permeability transition pore as a $\mathrm{Ca}^{2+}$ release channel: New answers to an old question. Cell Calcium 52: 22-27.

Bernardi P, Petronilli V, Di Lisa F, Forte M. 2001. A mitochondrial perspective on cell death. Trends Biochem Sci 26: $112-117$.

Bernardi P, Krauskopf A, Basso E, Petronilli V, BlachlyDyson E, Di Lisa F, Forte MA. 2006. The mitochondrial permeability transition from in vitro artifact to disease target. FEBS J 273: 2077-2099.

Bernardi P, Bonaldo P, Maraldi NM, Merlini L, Sabatelli P. 2009. On the pathogenesis of collagen VI muscular dystrophies-comment on article of Hicks et al. BRAIN 132: e121.

Bethlem J, Wijngaarden GK. 1976. Benign myopathy, with autosomal dominant inheritance. A report on three pedigrees. BRAIN 99: 91-100.

Bidanset DJ, Guidry C, Rosenberg LC, Choi HU, Timpl R, Hook M. 1992. Binding of the proteoglycan decorin to collagen type VI. J Biol Chem 267: 5250-5256.

Bonaldo P, Russo V, Bucciotti F, Bressan GM, Colombatti A. 1989. $\alpha 1$ Chain of chick type VI collagen. The complete cDNA sequence reveals a hybrid molecule made of one short collagen and three von Willebrand factor type Alike domains. J Biol Chem 264: 5575-5580.

Bonaldo P, Russo V, Bucciotti F, Doliana R, Colombatti A. 1990. Structural and functional features of the $\alpha 3$ chain indicate a bridging role for chicken collagen VI in connective tissues. Biochemistry 29: 1245-1254. 
Pathogenesis of Collagen VI Muscular Dystrophies

Bonaldo P, Braghetta P, Zanetti M, Piccolo S, Volpin D, Bressan GM. 1998. Collagen VI deficiency induces early onset myopathy in the mouse: An animal model for Bethlem myopathy. Hum Mol Genet 7: 2135-2140.

Bönnemann CG. 2011. The collagen VI-related myopathies: Muscle meets its matrix. Nat Rev Neurol 7: 379-390.

Braghetta P, Fabbro C, Piccolo S, Marvulli D, Bonaldo P, Volpin D, Bressan GM. 1996. Distinct regions control transcriptional activation of the $\alpha 1$ (VI) collagen promoter in different tissues of transgenic mice. J Cell Biol 135: 1163-1177.

Braghetta P, Ferrari A, Fabbro C, Bizzotto D, Volpin D, Bonaldo P, Bressan GM. 2008. An enhancer required for transcription of the Col6al gene in muscle connective tissue is induced by signals released from muscle cells. Exp Cell Res 314: 3508-3518.

Burg MA, Tillet E, Timpl R, Stallcup WB. 1996. Binding of the NG2 proteoglycan to type VI collagen and other extracellular matrix molecules. J Biol Chem 271: 2611026116.

Camacho Vanegas O, Bertini E, Zhang RZ, Petrini S, Minosse C, Sabatelli P, Giusti B, Chu ML, Pepe G. 2001. Ullrich scleroatonic muscular dystrophy is caused by recessive mutations in collagen type VI. Proc Natl Acad Sci 98: 7516-7521.

Carmignac V, Svensson M, Korner Z, Elowsson L, Matsumura C, Gawlik KI, Allamand V, Durbeej M. 2011. Autophagy is increased in laminin $\alpha 2$ chain-deficient muscle and its inhibition improves muscle morphology in a mouse model of MDC1A. Hum Mol Genet 20: 48914902.

Cereghetti GM, Stangherlin A, Martins de Brito O, Chang CR, Blackstone C, Bernardi P, Scorrano L. 2008. Dephosphorylation by calcineurin regulates translocation of Drp1 to mitochondria. Proc Natl Acad Sci 105: $15803-$ 15808.

Chu ML, Zhang RZ, Pan TC, Stokes D, Conway D, Kuo HJ, Glanville R, Mayer U, Mann K, Deutzmann R, et al. 1990. Mosaic structure of globular domains in the human type VI collagen $\alpha 3$ chain: Similarity to von Willebrand factor, fibronectin, actin, salivary proteins and aprotinin type protease inhibitors. EMBO J 9: 385-393.

Colombatti A, Bonaldo P. 1987. Biosynthesis of chick type VI collagen. II. Processing and secretion in fibroblasts and smooth muscle cells. J Biol Chem 262: 14461-14466.

Colombatti A, Bonaldo P. 1991. The superfamily of proteins with von Willebrand factor type A-like domains: One theme common to components of extracellular matrix, hemostasis, cellular adhesion, and defense mechanisms. Blood 77: 2305-2315.

Colombatti A, Bonaldo P, Ainger K, Bressan GM, Volpin D. 1987. Biosynthesis of chick type VI collagen. I. Intracellular assembly and molecular structure. J Biol Chem 262: 14454-14460.

Colombatti A, Mucignat MT, Bonaldo P. 1995. Secretion and matrix assembly of recombinant type VI collagen. J Biol Chem 270: 13105-13111.

Demir E, Sabatelli P, Allamand V, Ferreiro A, Moghadaszadeh B, Makrelouf M, Topaloglu H, Echenne B, Merlini L, Guicheney P. 2002. Mutations in COL6A3 cause severe and mild phenotypes of Ullrich congenital muscular dystrophy. Am J Hum Genet 70: 1446-1458.
Di Lisa F, Menabò R, Canton M, Barile M, Bernardi P. 2001. Opening of the mitochondrial permeability transition pore causes depletion of mitochondrial and cytosolic $\mathrm{NAD}^{+}$and is a causative event in the death of myocytes in postischemic reperfusion of the heart. J Biol Chem 276: 2571-2575.

Doliana R, Bonaldo P, Colombatti A. 1990. Multiple forms of chicken $\alpha 3$ (VI) collagen chain generated by alternative splicing in type A repeated domains. J Cell Biol 111: 2197-2205.

Foley AR, Hu Y, Zou Y, Columbus A, Shoffner J, Dunn DM, Weiss RB, Bonnemann CG. 2009. Autosomal recessive inheritance of classic Bethlem myopathy. Neuromuscul Disord 19: 813-817.

Furthmayr H, Wiedemann H, Timpl R, Odermatt E, Engel J. 1983. Electron-microscopical approach to a structural model of intima collagen. Biochem J 211: 303-311.

Galat A. 2003. Peptidylprolyl cis/trans isomerases (immunophilins): Biological diversity-targets-functions. Curr Top Med Chem 3: 1315-1347.

Gara SK, Grumati P, Urciuolo A, Bonaldo P, Kobbe B, Koch M, Paulsson M, Wagener R. 2008. Three novel collagen VI chains with high homology to the $\alpha 3$ chain. $J$ Biol Chem 283: 10658-10670.

Gara SK, Grumati P, Squarzoni S, Sabatelli P, Urciuolo A, Bonaldo P, Paulsson M, Wagener R. 2011. Differential and restricted expression of novel collagen VI chains in mouse. Matrix Biol 30: 248-257.

Grumati P, Coletto L, Sabatelli P, Cescon M, Angelin A, Bertaggia E, Blaauw B, Urciuolo A, Tiepolo T, Merlini L, et al. 2010. Autophagy is defective in collagen VI muscular dystrophies, and its reactivation rescues myofiber degeneration. Nat Med 16: 1313-1320.

Grumati P, Coletto L, Sandri M, Bonaldo P. 2011a. Autophagy induction rescues muscular dystrophy. Autophagy 7: 426-428.

Grumati P, Coletto L, Schiavinato A, Castagnaro S, Bertaggia E, Sandri M, Bonaldo P. 2011b. Physical exercise stimulates autophagy in normal skeletal muscles but is detrimental for collagen VI-deficient muscles. Autophagy 7: $1415-1423$.

Gualandi F, Urciuolo A, Martoni E, Sabatelli P, Squarzoni S, Bovolenta M, Messina S, Mercuri E, Franchella A, Ferlini A, et al. 2009. Autosomal recessive Bethlem myopathy. Neurology 73: 1883-1891.

Hicks D, Lampe AK, Laval SH, Allamand V, JimenezMallebrera C, Walter MC, Muntoni F, Quijano-Roy S, Richard P, Straub V, et al. 2009. Cyclosporine A treatment for Ullrich congenital muscular dystrophy: A cellular study of mitochondrial dysfunction and its rescue. BRAIN 132: 147-155.

Hüser J, Blatter LA. 1999. Fluctuations in mitochondrial membrane potential caused by repetitive gating of the permeability transition pore. Biochem J 343: $311-317$.

Irwin WA, Bergamin N, Sabatelli P, Reggiani C, Megighian A, Merlini L, Braghetta P, Columbaro M, Volpin D, Bressan GM, et al. 2003. Mitochondrial dysfunction and apoptosis in myopathic mice with collagen VI deficiency. Nat Genet 35: 267-271.

Jimenez-Mallebrera C, Maioli MA, Kim J, Brown SC, Feng L, Lampe AK, Bushby K, Hicks D, Flanigan KM, Bonnemann C, et al. 2006. A comparative analysis of collagen 
P. Bernardi and P. Bonaldo

VI production in muscle, skin and fibroblasts from 14 Ullrich congenital muscular dystrophy patients with dominant and recessive COL6A mutations. Neuromuscul Disord 16: 571-582.

Jöbsis GJ, Keizers H, Vreijling JP, de Visser M, Speer MC, Wolterman RA, Baas F, Bolhuis PA. 1996. Type VI collagen mutations in Bethlem myopathy, an autosomal dominant myopathy with contractures. Nat Genet 14: $113-$ 115.

Keene DR, Engvall E, Glanville RW. 1988. Ultrastructure of type VI collagen in human skin and cartilage suggests an anchoring function for this filamentous network. $J$ Cell Biol 107: 1995-2006.

Kuo HJ, Maslen CL, Keene DR, Glanville RW. 1997. Type VI collagen anchors endothelial basement membranes by interacting with type IV collagen. J Biol Chem 272: 26522-26529.

Lamande SR, Shields KA, Kornberg AJ, Shield LK, Bateman JF. 1999. Bethlem myopathy and engineered collagen VI triple helical deletions prevent intracellular multimer assembly and protein secretion. J Biol Chem 274: 2181721822.

Lamande SR, Morgelin M, Selan C, Jobsis GJ, Baas F, Bateman JF. 2002. Kinked collagen VI tetramers and reduced microfibril formation as a result of Bethlem myopathy and introduced triple helical glycine mutations. J Biol Chem 277: 1949-1956.

Lampe AK, Bushby KM. 2005. Collagen VI related muscle disorders. J Med Genet 42: 673-685.

Lampe AK, Zou Y, Sudano D, O'Brien KK, Hicks D, Laval SH, Charlton R, Jimenez-Mallebrera C, Zhang RZ, Finkel RS, et al. 2008. Exon skipping mutations in collagen VI are common and are predictive for severity and inheritance. Hum Mutat 29: 809-822.

Liu J, Farmer JDJ, Lane WS, Friedman J, Weissman I, Schreiber SL. 1991. Calcineurin is a common target of cyclophilin-cyclosporin A and FKBP-FK506 complexes. Cell 66: 807-815.

Lucioli S, Giusti B, Mercuri E, Vanegas OC, Lucarini L, Pietroni V, Urtizberea A, Ben Yaou R, de Visser M, van der Kooi AJ, et al. 2005. Detection of common and private mutations in the COL6A1 gene of patients with Bethlem myopathy. Neurology 64: 1931-1937.

Merlini L, Morandi L, Granata C, Ballestrazzi A. 1994. Bethlem myopathy: Early-onset benign autosomal dominant myopathy with contractures. Description of two new families. Neuromuscul Disord 4: 503-511.

Merlini L, Angelin A, Tiepolo T, Braghetta P, Sabatelli P, Zamparelli A, Ferlini A, Maraldi NM, Bonaldo P, Bernardi P. 2008a. Cyclosporin A corrects mitochondrial dysfunction and muscle apoptosis in patients with collagen VI myopathies. Proc Natl Acad Sci 105: 5225-5229.

Merlini L, Martoni E, Grumati P, Sabatelli P, Squarzoni S, Urciuolo A, Ferlini A, Gualandi F, Bonaldo P. 2008b. Autosomal recessive myosclerosis myopathy is a collagen VI disorder. Neurology 71: 1245-1253.

Merlini L, Sabatelli P, Armaroli A, Gnudi S, Angelin A, Grumati P, Michelini ME, Franchella A, Gualandi F, Bertini E, et al. 2011. Cyclosporine A in Ullrich congenital muscular dystrophy: Long-term results. Oxid Med Cell Longev 2011: 139194.
Millay DP, Sargent MA, Osinska H, Baines CP, Barton ER, Vuagniaux G, Sweeney HL, Robbins J, Molkentin JD. 2008. Genetic and pharmacologic inhibition of mitochondrial-dependent necrosis attenuates muscular dystrophy. Nat Med 14: 442-447.

Mizushima N, Levine B, Cuervo AM, Klionsky DJ. 2008. Autophagy fights disease through cellular self-digestion. Nature 451: 1069-1075.

Palma E, Tiepolo T, Angelin A, Sabatelli P, Maraldi NM, Basso E, Forte MA, Bernardi P, Bonaldo P. 2009. Genetic ablation of cyclophilin D rescues mitochondrial defects and prevents muscle apoptosis in collagen VI myopathic mice. Hum Mol Genet 18: 2024-2031.

Pan TC, Zhang RZ, Sudano DG, Marie SK, Bonnemann CG, Chu ML. 2003. New molecular mechanism for Ullrich congenital muscular dystrophy: A heterozygous in-frame deletion in the COL6Al gene causes a severe phenotype. Am J Hum Genet 73: 355-369.

Pepe G, Bertini E, Bonaldo P, Bushby K, Giusti B, de Visser M, Guicheney P, Lattanzi G, Merlini L, Muntoni F, et al. 2002. Bethlem myopathy (BETHLEM) and Ullrich scleroatonic muscular dystrophy: 100th ENMC international workshop, 23-24 November 2001, Naarden, The Netherlands. Neuromuscul Disord 12: 984-993.

Pepe G, Lucarini L, Zhang RZ, Pan TC, Giusti B, QuijanoRoy S, Gartioux C, Bushby KM, Guicheney P, Chu ML. 2006. COL6A1 genomic deletions in Bethlem myopathy and Ullrich muscular dystrophy. Ann Neurol 59: 190195.

Petronilli V, Nicolli A, Costantini P, Colonna R, Bernardi P. 1994. Regulation of the permeability transition pore, a voltage-dependent mitochondrial channel inhibited by cyclosporin A. Biochim Biophys Acta 1187: 255-259.

Petronilli V, Miotto G, Canton M, Brini M, Colonna R, Bernardi P, Di Lisa F. 1999. Transient and long-lasting openings of the mitochondrial permeability transition pore can be monitored directly in intact cells by changes in mitochondrial calcein fluorescence. Biophys $J$ 76: 725-734.

Petronilli V, Penzo D, Scorrano L, Bernardi P, Di Lisa F. 2001. The mitochondrial permeability transition, release of cytochrome $c$ and cell death. Correlation with the duration of pore openings in situ. J Biol Chem 276: 12030-12034.

Pfaff M, Aumailley M, Specks U, Knolle J, Zerwes HG, Timpl R. 1993. Integrin and Arg-Gly-Asp dependence of cell adhesion to the native and unfolded triple helix of collagen type VI. Exp Cell Res 206: 167-176.

Porcelli AM, Angelin A, Ghelli A, Mariani E, Martinuzzi A, Carelli V, Petronilli V, Bernardi P, Rugolo M. 2008. Respiratory complex I dysfunction due to mitochondrial DNA mutations shifts the voltage threshold for opening of the permeability transition pore toward resting levels. J Biol Chem 284: 2045-2052.

Porcelli AM, Angelin A, Ghelli A, Mariani E, Martinuzzi A, Carelli V, Petronilli V, Bernardi P, Rugolo M. 2009. Respiratory complex I dysfunction due to mitochondrial DNA mutations shifts the voltage threshold for opening of the permeability transition pore toward resting levels. J Biol Chem 284: 2045-2052.

Rasola A, Bernardi P. 2011. Mitochondrial permeability transition in $\mathrm{Ca}^{2+}$-dependent apoptosis and necrosis. Cell Calcium 50: 222-233. 
Reutenauer J, Dorchies OM, Patthey-Vuadens O, Vuagniaux G, Ruegg UT. 2008. Investigation of Debio 025, a cyclophilin inhibitor, in the dystrophic mdx mouse, a model for Duchenne muscular dystrophy. Br J Pharmacol 155: 574-584.

Sabatelli P, Bonaldo P, Lattanzi G, Braghetta P, Bergamin N, Capanni C, Mattioli E, Columbaro M, Ognibene A, Pepe G, et al. 2001. Collagen VI deficiency affects the organization of fibronectin in the extracellular matrix of cultured fibroblasts. Matrix Biol 20: 475-486.

Sabatelli P, Gara SK, Grumati P, Urciuolo A, Gualandi F, Curci R, Squarzoni S, Zamparelli A, Martoni E, Merlini L, et al. 2011. Expression of the collagen VI $\alpha 5$ and $\alpha 6$ chains in normal human skin and in skin of patients with collagen VI-related myopathies. J Invest Dermatol 131: 99-107.

Sabatelli P, Palma E, Angelin A, Squarzoni S, Urciuolo A, Pellegrini C, Tiepolo T, Bonaldo P, Gualandi F, Merlini L, et al. 2012. Critical evaluation of the use of cell cultures for inclusion in clinical trials of patients affected by collagen VI myopathies. J Cell Physiol 227: 2927-2935.

Sasaki T, Hohenester E, Zhang RZ, Gotta S, Speer MC, Tandan R, Timpl R, Chu ML. 2000. A Bethlem myopathy Gly to Glu mutation in the von Willebrand factor A domain $\mathrm{N} 2$ of the collagen $\alpha 3(\mathrm{VI})$ chain interferes with protein folding. FASEB J 14: 761-768.

Scacheri PC, Gillanders EM, Subramony SH, Vedanarayanan V, Crowe CA, Thakore N, Bingler M, Hoffman EP. 2002. Novel mutations in collagen VI genes: Expansion of the Bethlem myopathy phenotype. Neurology 58: 593-602.

Schessl J, Zou Y, Bonnemann CG. 2006. Congenital muscular dystrophies and the extracellular matrix. Semin Pediatr Neurol 13: 80-89.

Scorrano L, Ashiya M, Buttle K, Weiler S, Oakes SA, Mannella CA, Korsmeyer SJ. 2002. A distinct pathway remodels mitochondrial cristae and mobilizes cytochrome $c$ during apoptosis. Dev Cell 2: 55-67.

Squarzoni S, Sabatelli P, Bergamin N, Guicheney P, Demir E, Merlini L, Lattanzi G, Ognibene A, Capanni C, Mat- tioli E, et al. 2006. Ultrastructural defects of collagen VI filaments in an Ullrich syndrome patient with loss of the $\alpha 3$ (VI) N10-N7 domains. J Cell Physiol 206: 160166.

Telfer WR, Busta AS, Bonnemann CG, Feldman EL, Dowling JJ. 2010. Zebrafish models of collagen VI-related myopathies. Hum Mol Genet 19: 2433-2444.

Tiepolo T, Angelin A, Palma E, Sabatelli P, Merlini L, Nicolosi L, Finetti F, Braghetta P, Vuagniaux G, Dumont JM, et al. 2009. The cyclophilin inhibitor Debio 025 normalizes mitochondrial function, muscle apoptosis and ultrastructural defects in Col6a1 ${ }^{-/-}$myopathic mice. $\mathrm{Br} \mathrm{J}$ Pharmacol 157: 1045-1052.

Tolkovsky AM. 2010. Autophagy thwarts muscle disease. Nat Med 16: 1188-1190.

Tooley LD, Zamurs LK, Beecher N, Baker NL, Peat RA, Adams NE, Bateman JF, North KN, Baldock C, Lamande SR. 2010. Collagen VI microfibril formation is abolished by an $\alpha 2$ (VI) von Willebrand factor type A domain mutation in a patient with Ullrich congenital muscular dystrophy. J Biol Chem 285: 33567-33576.

Vinogradov A, Scarpa A, Chance B. 1972. Calcium and pyridine nucleotide interaction in mitochondrial membranes. Arch Biochem Biophys 152: 646-654.

Wiberg C, Hedbom E, Khairullina A, Lamande SR, Oldberg A, Timpl R, Morgelin M, Heinegard D. 2001. Biglycan and decorin bind close to the N-terminal region of the collagen VI triple helix. J Biol Chem 276: 1894718952.

Zhang RZ, Sabatelli P, Pan TC, Squarzoni S, Mattioli E, Bertini E, Pepe G, Chu ML. 2002. Effects on collagen VI mRNA stability and microfibrillar assembly of three COL6A2 mutations in two families with Ullrich congenital muscular dystrophy. J Biol Chem 277: 43557-43564.

Zou Y, Zhang RZ, Sabatelli P, Chu ML, Bonnemann CG. 2008. Muscle interstitial fibroblasts are the main source of collagen VI synthesis in skeletal muscle: Implications for congenital muscular dystrophy types Ullrich and Bethlem. J Neuropathol Exp Neurol 67: 144-154. 


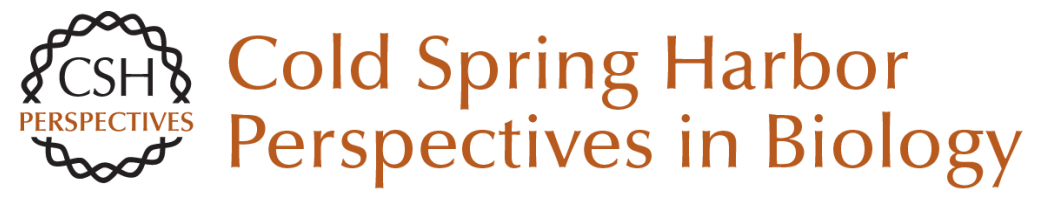

\title{
Mitochondrial Dysfunction and Defective Autophagy in the Pathogenesis of Collagen VI Muscular Dystrophies
}

\author{
Paolo Bernardi and Paolo Bonaldo
}

Cold Spring Harb Perspect Biol 2013; doi: 10.1101/cshperspect.a011387 originally published online April 11,2013

\section{Subject Collection Mitochondria}

Altered Sulfide $\left(\mathrm{H}_{2} \mathrm{~S}\right)$ Metabolism in Ethylmalonic Encephalopathy Valeria Tiranti and Massimo Zeviani

Mitochondrial DNA Genetics and the Heteroplasmy Conundrum in Evolution and Disease Douglas C. Wallace and Dimitra Chalkia

The Role of Mitochondria in Cellular Iron-Sulfur Protein Biogenesis: Mechanisms, Connected Processes, and Diseases Oliver Stehling and Roland Lill

Mechanisms of Mitochondrial Fission and Fusion Alexander M. van der Bliek, Qinfang Shen and Sumihiro Kawajiri

The Mitochondrial Nucleoid: Integrating Mitochondrial DNA into Cellular Homeostasis Robert Gilkerson, Liliana Bravo, Iraselia Garcia, et al.

Relevance of Mitochondrial Genetics and Metabolism in Cancer Development Giuseppe Gasparre, Anna Maria Porcelli, Giorgio Lenaz, et al.

Mitochondrial Quality Control Mediated by PINK1 and Parkin: Links to Parkinsonism

Derek Narendra, John E. Walker and Richard Youle
Where Killers Meet--Permeabilization of the Outer Mitochondrial Membrane during Apoptosis Tom Bender and Jean-Claude Martinou

Mitochondrial Biogenesis through Activation of Nuclear Signaling Proteins John E. Dominy and Pere Puigserver

Mitochondrial Trafficking in Neurons Thomas L. Schwarz

Mitochondrial Dysfunction and Defective Autophagy in the Pathogenesis of Collagen VI Muscular Dystrophies Paolo Bernardi and Paolo Bonaldo

Clinical and Molecular Features of POLG-Related Mitochondrial Disease Jeffrey D. Stumpf, Russell P. Saneto and William $C$. Copeland

Mitochondrial Metabolism, Sirtuins, and Aging Michael N. Sack and Toren Finkel

Mechanisms of Protein Sorting in Mitochondria Diana Stojanovski, Maria Bohnert, Nikolaus Pfanner, et al.

For additional articles in this collection, see http://cshperspectives.cshlp.org/cgi/collection/

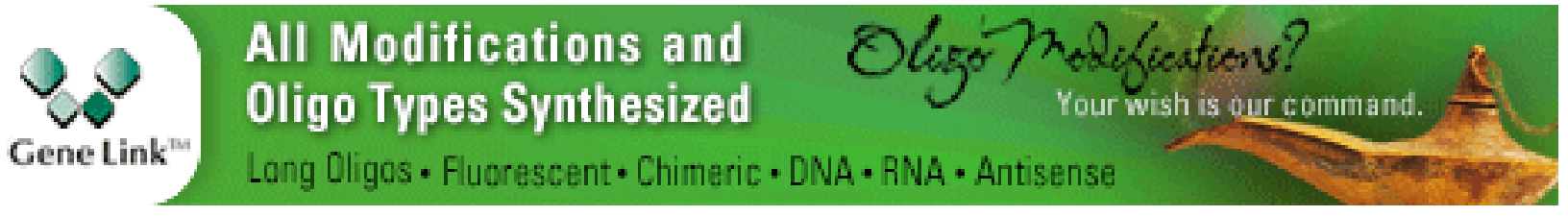




\section{Mitochondrial Evolution}

Michael W. Gray

For additional articles in this collection, see http://cshperspectives.cshlp.org/cgi/collection/

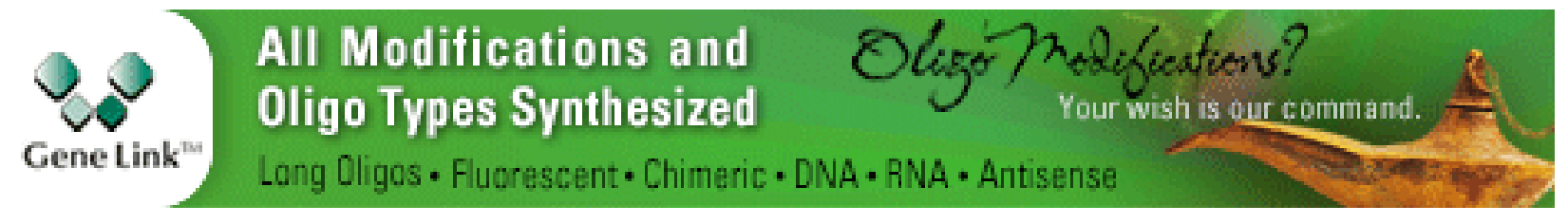

Copyright @ 2013 Cold Spring Harbor Laboratory Press; all rights reserved 